\title{
Molecular dynamics of the immune checkpoint programmed cell death protein I, PD-1: conformational changes of the BC- loop upon binding of the ligand PD-L1 and the monoclonal antibody nivolumab
}

\author{
Bernhard Roither ${ }^{1}$, Chris Oostenbrink ${ }^{2}$ and Wolfgang Schreiner ${ }^{1 *}$ (B)
}

From 3rd International Workshop on Computational Methods for the Immune System Function (CMISF 2019) San Diego, CA, USA. 18-21 November 2019

\section{*Correspondence:}

wolfgang.

schreiner@meduniwien.ac.at

${ }^{1}$ Institute of Biosimulation

and Bioinformatics, Medical

University of Vienna,

Spitalgasse 23/88.04.510,

1090 Vienna, Austria

Full list of author information is available at the end of the article

\begin{abstract}
Background: The immune checkpoint receptor programmed cell death protein I (PD-1) has been identified as a key target in immunotherapy. PD-1 reduces the risk of autoimmunity by inducing apoptosis in antigen-specific T cells upon interaction with programmed cell death protein ligand I (PD-L1). Various cancer types overexpress PD-L1 to evade the immune system by inducing apoptosis in tumor-specific CD8+ T cells. The clinically used blocking antibody nivolumab binds to PD-1 and inhibits the immunosuppressive interaction with PD-L1. Even though PD-1 is already used as a drug target, the exact mechanism of the receptor is still a matter of debate. For instance, it is hypothesized that the signal transduction is based on an active conformation of PD-1.
\end{abstract}

Results: Here we present the results of the first molecular dynamics simulations of PD-1 with a complete extracellular domain with a focus on the role of the BC-loop of PD-1 upon binding PD-L1 or nivolumab. We could demonstrate that the BC-loop can form three conformations. Nivolumab binds to the BC-loop according to the conformational selection model whereas PD-L1 induces allosterically a conformational change of the BC-loop.

Conclusion: Due to the structural differences of the BC-loop, a signal transduction based on active conformation cannot be ruled out. These findings will have an impact on drug design and will help to refine immunotherapy blocking antibodies.

Keywords: PD-1, PD-L1, Nivolumab, Molecular dynamics, BC-loop, Mathematical oncology source, provide a link to the Creative Commons licence, and indicate if changes were made. The images or other third party material in this article are included in the article's Creative Commons licence, unless indicated otherwise in a credit line to the material. If material is not included in the article's Creative Commons licence and your intended use is not permitted by statutory regulation or exceeds the permitted use, you will need to obtain permission directly from the copyright holder. To view a copy of this licence, visit http://creativecommons.org/ licenses/by/4.0/. The Creative Commons Public Domain Dedication waiver (http://creativecommons.org/publicdomain/zero/1.0/) applies to the data made available in this article, unless otherwise stated in a credit line to the data. 


\section{Background}

Programmed cell death protein I (PD-1) is a type 1 transmembrane protein in mainly $\mathrm{T}$ and $\mathrm{B}$ cells and a so-called immune checkpoint as it promotes self-tolerance by inducing apoptosis of antigen-specific $\mathrm{T}$ cells, a mechanism which is often exploited by cancer cells. Thus, PD-1 has recently emerged as key target in cancer immunotherapy.

The regulatory effect of PD-1 is triggered upon binding the cell death protein I ligand 1 (PD-L1) which is mainly expressed on macrophages, dendritic cells and a variety of tissue cells [1]. Naïve T-cells reside in the lymph nodes and become activated upon interaction of the T cell receptor (TCR) with antigen-presenting cells (APC) which display antigens via the major histocompatibility complex (MHC) II. However, secondary co-stimulation of PD-1 with APC PD-L1 results in the T cell apoptosis instead [2]. The interaction between PD-1 and PD-L1 can take place in all stages of the T cell lifespan. Occurrences ranging from the early stage of $\mathrm{T}$ cell activation to the operational inflamed tissue site manifest the importance of the PD-1/PD-L1 pathway for the tight regulation of the immune system [3]. Cancer cells which express PD-L1 evade the host immune system by inducing apoptosis in cancer antigen specific T cells [4]. In a new approach, blocking antibodies that either target PD-1 or PD-L1 are used to disable the interaction between PD-1 and PD-L1. Thus, the T cells stay active and can target the cancer cells. Recently the PD-1 blocking antibody nivolumab was approved by the FDA. Nivolumab is clinically used for the treatment of melanoma, metastatic renal cell carcinomas, classical Hodgkin lymphoma (cHl) and non-small-cell lung carcinoma (NSCLC) [5].

Even though PD-1 is already successfully used as an immunotherapy drug target the exact mechanism of the receptor is not fully understood yet [6]. For instance, the signal transduction from the extracellular domain into the intracellular domain is still a matter of debate, enriched by modelling approaches [7-9]. Presumably, the binding of PD-L1 induces an active conformation in PD-1 which facilitates the PD-1 signaling cascade inside the $\mathrm{T}$ cell. Indeed, conformational changes upon ligand binding were discovered [10]. In a molecular dynamics (MD) simulation the switch from the open to closed CC'-loop conformation of PD-1 upon PD-L1 binding was described [11]. In other MD simulations the influence of mutations on ligand binding was examined [12, 13]. Also the conformational dynamics of PD-L1 were studied with MD [14, 15]. We have also performed preliminary investigations [16] which are supplemented in the current work.

In a similar approach, we examine the conformational changes of the BC-loop upon binding either PD-L1 or nivolumab. MD studies of PD-1 unbound and bound to PD-L1 and the clinically used antibody nivolumab were performed. This provided insight into flexibility of residues and conformational movements of the BC-loop which may be important for the optimization of already existing PD-1 antibodies or the design of new antibodies or small molecular compounds.

\section{Methods}

The following notation will be used: PD-1 unbound, PD-1 - PD-L1 and PD-1 nivolumab when the whole simulated system is addressed. PD- $1_{\mathrm{Apo}}, \mathrm{PD}-1_{\mathrm{PD}-\mathrm{L} 1}$ and PD- $1_{\text {Niv }}$ refer specifically to PD- 1 within the respective simulated system. 


\section{Preprocessing}

The crystal structures PD-1 unbound (3RRQ), PD-1 - PD-L1 (4ZQK) and PD-1 nivolumab (5WT9) were used for simulations. With each binding partner different parts of PD-1 crystallized and therefore the files had to be manually curated first. Missing atoms and single residues were added with Swiss PDB-Viewer. The C'D-loop (residues 65-92) was taken from PD-1 - pembrolizumab (5GGS) and added to each system. The N-loop (25-34) was taken from 5WT9 and added to 3RRQ and 4ZQK. The PD-1 structures of each PDB file were aligned with VMD to orientate them in the same direction. The missing loops were copied and pasted into the PDB files. Hence, each PD-1 included the residues from 25 to 149 which represent the complete extracellular domain.

\section{Simulation}

MD simulations were performed on a node of the Vienna Scientific Cluster (VSC) consisting of two processors (Intel Xeon E5-2650v2, 2.6GHz, 8 cores from Ivy Bridge-EP family) and a GPU (NVIDIA Pascal GeForce GTX 1080) with GROMACS 2018.1 software package [17]. The 2018.1 version has an improved performance because long-ranged non-bonded interactions can be computed on a single GPU. Also, it offers greater control on the usage of the GPUs. The GROMOS 54A7 force field was used to generate the topologies [18]. The version 54A7 was published in 2011 and includes four main improvements: new $\phi / \psi$ torsional angle terms were introduced, new atom type for a charged $-\mathrm{CH} 3$ in the choline moiety was added, to reproduce free energy of hydration $\mathrm{Na}^{+}$and $\mathrm{Cl}^{-}$ions were modified and additional torsional angles were included. Overall, the GROMOS 54A7 force field has improved stability of secondary structures [18]. Proteins were solvated in a cubic box with SPC water and a minimum distance to the box edge of $1.0 \mathrm{~nm}[19] . \mathrm{Na}^{+}$and $\mathrm{Cl}^{-}$were added to neutralize the net charges. For energy minimization the steepest descent minimization algorithm with a step size of $0.01 \mathrm{~nm}$ and a maximum of 50,000 steps was applied and then stopped once the maximum force was smaller than $0.1 \mathrm{~kJ} \mathrm{~mol}^{-1} \mathrm{~nm}^{-1}$. For neighbor search, the Verlet cut-off scheme of $1.4 \mathrm{~nm}$ was used. To calculate the electrostatic forces, the particle mesh Ewald algorithm with a cut-off of $1.4 \mathrm{~nm}$ was applied. For the Van der Waals forces a cut-off of $1.4 \mathrm{~nm}$ was adopted. The NVT and NPT equilibration runs took $0.1 \mathrm{~ns}$ with $5 \times 10^{4}$ steps and a step size of $2 \mathrm{fs}$. The production runs were carried out for $100 \mathrm{~ns}$ and $10 \times 10$ ns with $5 \times 10^{7}$ steps with a step size of $2 \mathrm{fs}$. Temperature and pressure coupling were set to $300 \mathrm{~K}$ (velocity rescaling) and 1 bar (Berendsen), respectively. All bonds were constraint to their optimal length using the LINCS algorithm. Energies and coordinates were saved every 10 ps.

\section{Analysis}

\section{RMSF}

The root-mean-square fluctuation (RMSF) of atomic positions (i.e. standard deviation) gives the displacement of an atom at position $\mathbf{x}$ with respect to its time-averaged position $\overline{\mathbf{x}}$. $\mathrm{C} \alpha$ atoms were used for least-square fitting of the trajectories to the starting structure and RMSF calculations:

$$
\operatorname{RMSF}(\mathbf{x})=\sqrt{\frac{1}{\mathrm{~T}} \sum_{\mathrm{i}=1}^{\mathrm{T}}\left\|\mathbf{x}_{\mathrm{i}}\left(\mathrm{t}_{\mathrm{i}}\right)-\overline{\mathbf{x}}\right\|^{2}}
$$


where $T$ is the total number of time steps within the respective trajectory. To calculate the RMSF, the ten $10 \mathrm{~ns}$ long trajectories were combined and treated as if they were a single $100 \mathrm{~ns}$ long trajectory.

\section{RMSD}

The root-mean-square deviation (RSMD) of atomic positions with the BC-loop was calculated after a least-square fit of the $C_{\alpha}$ backbone of the flanking regions. The RMSD is calculated at a time $t$ with respect to a given reference structure at time $t_{\text {ref: }}$

$$
\operatorname{RMSD}\left(\mathrm{t}_{\text {ref }}, \mathrm{t}\right)=\sqrt{\frac{1}{\mathrm{~N}} \sum_{\mathrm{i}=1}^{\mathrm{N}}\left\|\mathbf{x}_{\mathrm{i}}(\mathrm{t})-\mathbf{x}_{i}\left(\mathrm{t}_{\mathrm{ref}}\right)\right\|^{2}}
$$

where $\mathbf{x}_{i}(t)$ is the position of atom $i$ at time $t$ and $N$ is the total number of atoms in that part of the structure to which the RMSD refers, in that case the BC-loop.

\section{Clustering}

Based on RMSD structures were clustered as described by Daura et al. [20] which consists of the following steps:

1. Define each structure as cluster center

2. Count number of structures within defined cut-off (here $0.2 \mathrm{~nm}$ was set) i.e. neighbors

3. Select center with most neighbors, designate it as a cluster and remove set of structures from matrix

4. Repeat until all structures have been assigned to a cluster

Of the biggest 25 clusters the central structures were subjected to non-metric multidimensional scaling to display the structures in a representative two-dimensional space [21]:

1. Choose a random configuration of points in the two-dimensional space

2. Calculate distances between these points

3. Arrange points to maximize rank-order correlation between original RMSD matrix and new space distance

4. Calculate stress and compare to Kruskal's normalized convergence criterion. If convergence criterion is fulfilled exit, else return to 2 .

\section{Hydrogen bonds}

The hydrogen bonds were determined with GROMACS 2018.1 software package according to the distance and angle of hydrogen donors and acceptors. By default $-\mathrm{OH}$ and $-\mathrm{NH}$ groups were regarded as donors and $-\mathrm{O}$ and $-\mathrm{N}$ as acceptors. Hydrogen-donoracceptor angle and distance cut-offs were set to $30^{\circ}$ and $0.35 \mathrm{~nm}$, respectively. Donors and acceptors within that threshold were considered to form hydrogen bonds. 


\section{Non-bonded interactions}

The non-bonded interactions comprise electrostatic and Van der Waals (VdW) interactions. The electrostatic interactions arise from the unequal distribution of charges in molecules and are given by the Coulomb potential $\mathrm{E}_{\mathrm{Coul}}$. VdW interactions are a combination of dispersion, repulsion and induction forces and are given by the Lennard-Jones (LJ) potential $\mathrm{E}_{\mathrm{LJ}}$. To compute $\mathrm{E}_{\mathrm{Coul}}$ and $\mathrm{E}_{\mathrm{LJ}}$ with Gromacs 2018.1 a simulation rerun of the existing trajectories was invoked. The short-range Coulomb and LJ energies were extracted and summed up.

\section{Results and discussion}

Three different systems, PD-1 unbound, PD-1 - PD-L1 and PD-1 - nivolumab, were each simulated for $100 \mathrm{~ns}$ and $10 \times 10 \mathrm{~ns}$. Every $10 \mathrm{ps}$ the coordinates of atoms were saved. The structures of PD-1 of each system were extracted and timewise concatenated. RMSF and RMSD were calculated for the BC-loop and the Daura et al. [20] clustering algorithm was performed. Central structures of each cluster, as identified by the Daura algorithm, were then subjected to multidimensional scaling to identify common conformations of the BC-loop of PD- 1 across different binding states.

The RMSF (Fig. 1) illustrates the influence of the ligands on the flexibility of the PD-1 loops. In $\beta$-sheet regions the RMSF of PD-1 (independent of the binding partner) is around $0.1 \mathrm{~nm}$, whereas in areas of loops the values differ considerably. In the $\mathrm{CC}^{\prime}$-loop, it has been described that PD-1 $1_{\mathrm{Apo}}$ switches between the open and closed conformation whereas PD-L1 shifts the equilibrium towards the closed conformation [11]. Our results confirm these finding as, the RMSF of PD-1 $1_{\mathrm{PD}-\mathrm{L} 1}$ in the CC'-loop is lower than in PD- $1_{\mathrm{Apo}}$. Furthermore, nivolumab binding was found to decrease the flexibility of the N-loop. This is plausible, as it is the only ligand that directly interacts with the N-loop [22]. The C'D-loop could so far only be crystalized when stabilized with pembrolizumab [22]. Here, the C'D-loop was added from the

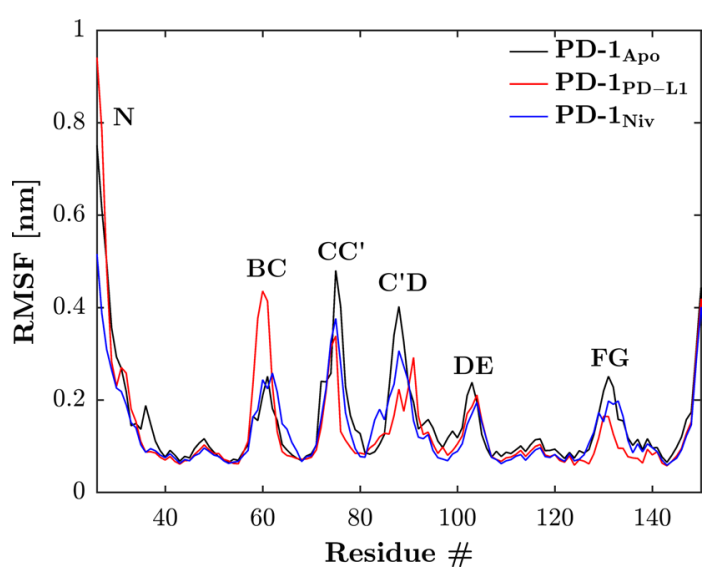

Fig. 1 Binding partners influence flexibility of loops. The RMSF of $C_{a} s$ of PD-1 unbound (black) and bound to either PD-L1 (red) or nivolumab (blue), when fitted to its respective first frame are shown. Whereas the impact of the binding partners on structured domains is negligible, the RMSF of the loops can change drastically. PD- $1_{\text {Apo }}$ has the greatest flexibility except for the BC-loop. The fact that PD-L1 seems to induce more flexibility in the BC-loop led to detailed examination of the BC-loop 
PD-1 - pembrolizumab system (5GGS) to complete the extracellular domain of PD-1. Even though both PD-L1 and nivolumab do not directly interact with the C'D-loop they still decrease the flexibility of the C'D-loop. Except for the BC-loop, the RMSF is highest in PD-1 $1_{\mathrm{Apo}}$ across all domains. Again, PD-L1 does not directly interact with the BC-loop, but whereas PD-L1 still decreases the flexibility in the C'D-loop the opposite occurs in the $\mathrm{BC}$-loop. $\mathrm{PD}-\mathrm{L} 1$ increases the flexibility of the $\mathrm{BC}$-loop significantly. Therefore, the BC-loop was further examined.

More than $90 \%$ of the structures of PD- $1_{\mathrm{Apo}}$ distribute between cluster 2 and 7 in a ratio of 82.5 to $10 \%$, respectively when clustering is based on the RMSD of the BCloop (Fig. 2a). Two point five percent of the PD- $1_{\text {Apo }}$ structures fall into cluster 9 . Cluster 1 consists of structures of both, PD-1 $1_{\mathrm{PD}-\mathrm{L} 1}$ and PD- $1_{\mathrm{Niv}}$. Twenty-five and sixty percent of the time the $\mathrm{BC}$-loop of PD- $1_{\mathrm{PD}-\mathrm{L} 1}$ and $\mathrm{PD}-1_{\mathrm{Niv}}$ exhibit the same conformation, respectively. Clusters 3 and 5 do not show any overlaps and contains only structures of PD- $1_{\mathrm{PD}-\mathrm{L} 1}$. Cluster 4 consists primarily of structures of PD- $1_{\text {Niv }}$, he structures of PD- $1_{\mathrm{Apo}}$ and PD-1 $1_{\mathrm{PD}-\mathrm{L} 1}$ are negligible $(<1 \%)$. Cluster 6 and 8 show again overlaps and contain structures of PD- $1_{\mathrm{PD}-\mathrm{L} 1}$ as well as PD- $1_{\mathrm{Niv}}$. The impact of different cutoffs on clustering is shown in supplementary Figure 1: Larger cut-off allows for more overlaps whereas smaller cutoff yields homogenous clusters, containing configurations from one system only.

The distance map (Fig. 2b) generated with multidimensional scaling [21] shows the relationship between the clusters found with the Daura et al. [20] clustering algorithm. The central frames of each cluster were scaled into the two-dimensional space and clusters of similar structure appear in proximity. Three distinct areas emerged and were named meta-cluster I, II and III. Meta-cluster I consists of clusters of all three systems, whereas meta-cluster II and III only consist of clusters from PD- $1_{\text {Apo }}$ and PD- $1_{\mathrm{PD}-\mathrm{L} 1}$, respectively. Eighty percent of the time, the $\mathrm{BC}$-loop of $\mathrm{PD}-1_{\mathrm{Apo}}$ resides in the meta-cluster II. PD- $1_{\mathrm{PD}-\mathrm{L} 1}$ resides $30 \%$ of the time in the meta-cluster III. The BC-loop of PD- $1_{\mathrm{Apo}}$, PD- $1_{\mathrm{PD}-\mathrm{L} 1}$ and PD-1 $1_{\mathrm{Niv}}$ reside 20,70 and $100 \%$ of the time in meta-cluster I. The results of the distance map suggest that the BC-loop can exhibit three conformations. Indeed, visualization with VMD 19.3 of the structures of the central frames of the clusters 1 to 5 show three distinct conformations (Fig. 2c). The BC-loop of cluster 1, 4 and 5 (all part of meta-cluster I) appear as bundle which is oriented towards the N-terminal end of PD-1. Cluster 2 (part of meta-cluster II) and cluster 3 (meta-cluster III) show distinct conformations. The BC-loop of the central structure of cluster 3 appears oriented to the center of PD-1. The structures of cluster 2 are in between the bundle of clusters 1,4 , and 5 and cluster 3. Based on the visualization of the central frames it is concluded that multidimensional scaling is a well-suited method to preselect frames to identify conformations with a molecular visualization program.

To investigate convergence behavior, we generated clusters not only for the whole $100 \mathrm{~ns}$ trajectory but for increasing parts of it, i.e. the first $10 \mathrm{~ns}, 20 \mathrm{~ns}, 30 \mathrm{~ns}$ etc., with a constant cut-off of $0.2 \mathrm{~nm}$. As the simulation samples increasing percentage of phase space, the numbers of clusters increase (Supplement Figure 2). Around $70 \mathrm{~ns}$ the number of clusters of the $\mathrm{BC}$-loop of the PD- $1_{\mathrm{Apo}}$ simulation seems to level off at 18 clusters. This indicates sufficient sampling over configuration space and that the system reaches convergence within $100 \mathrm{~ns}$. 

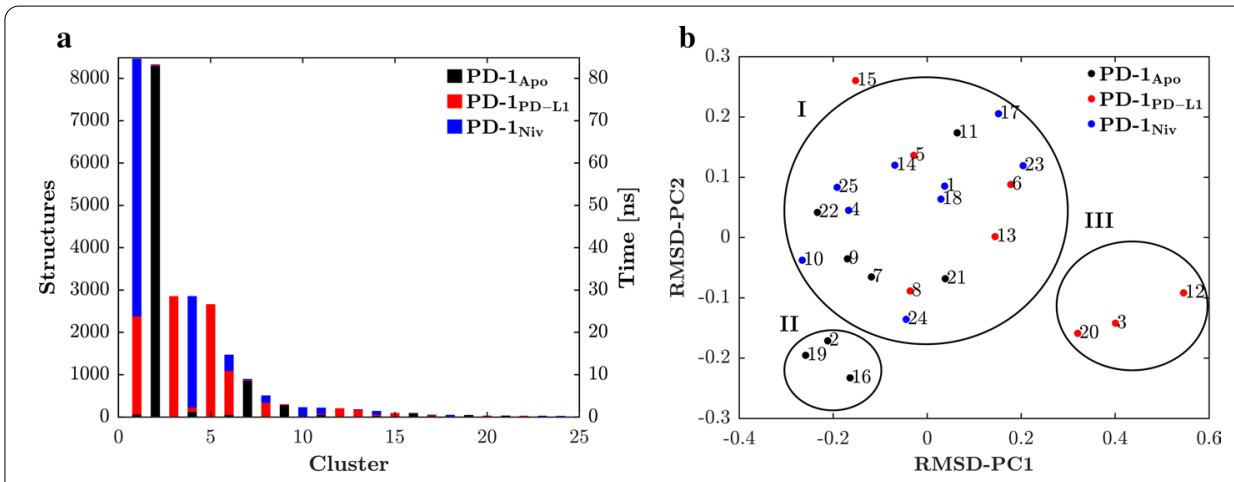

c

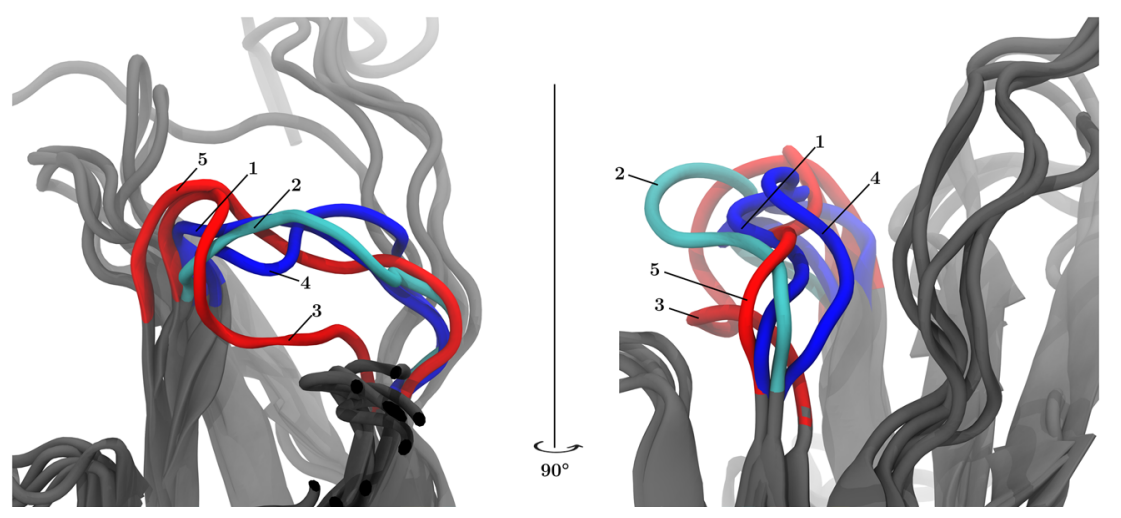

Fig. 2 PD-L1 induces a unique BC-loop conformation. The clustering algorithm as described by Daura et al. [20] was applied (a). Colors denote the origin of the structures. Only the first 25 clusters are shown. PD-1 PD-L1 $_{\text {(red) and PD-1 }}$ Niv (blue) exhibit for 25 and $60 \%$ of the time, respectively the same conformation. Over $90 \%$ of the structures of PD-1 ${ }_{\text {Apo }}$ (black) are distributed between cluster 2 and 7 . Cluster 3 and 5 contain only PD-1 PD-L1 $_{1}$ structures whereas cluster 4 consist primarily PD-1 ${ }_{\text {Niv }}$ structures. The number of structures is equivalent to the time the $\mathrm{BC}$-loop resided in a certain conformation. $\mathbf{b}$ The distance map generated with multidimensional scaling [21] shows the relationship between the conformations found with the Daura et al. [20] clustering algorithm. Clusters with similar structures are in proximity. Three areas are distinguishable (circled by hand) which are named meta-cluster I, II and III. Meta-cluster I is occupied by the PD- ${ }_{\text {Apo' }}$

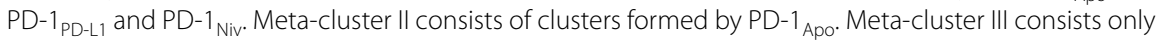

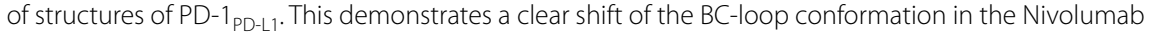
complex. The colors represent the origins of the central frames. c Superimposed crystal structures of the central frames of the first five clusters visualized with VMD 1.9.3. Structures of clusters 1,4 and 5 exhibit a similar BC-loop conformation. Cluster 2 and 3 exhibit distinct BC-loop conformations. BC-loop is colored; other domains are shown in grey. PD-1 ${ }_{\text {Apo }}$ structure is shown in cyan instead of black

Furthermore, the results suggest a complex mechanism of conformational changes of the BC-loop. Depending on the binding partner the conformational change of the $\mathrm{BC}$ loop is based on the conformational selection or the induced fit model [23]. Both concepts assume a conformational change of the unbound (apo-)protein to bind a ligand. The fundamental difference is whether the conformational change happens before or after ligand binding. In the induced-fit model the partner induces the active binding conformation of the protein. On the hand, in the conformational-selection model the active and inactive forms coexist even in absence of a ligand, but the ligand shifts the equilibrium to the active conformation [24]. The BC-loop exists in PD- $1_{\mathrm{Apo}}$ to $20 \%$ in conformation I and to $80 \%$ in conformation II. Upon binding to nivolumab the equilibrium is shifted to $100 \%$ of conformation I. PD-L1, on the other hand, induces a BC-loop 

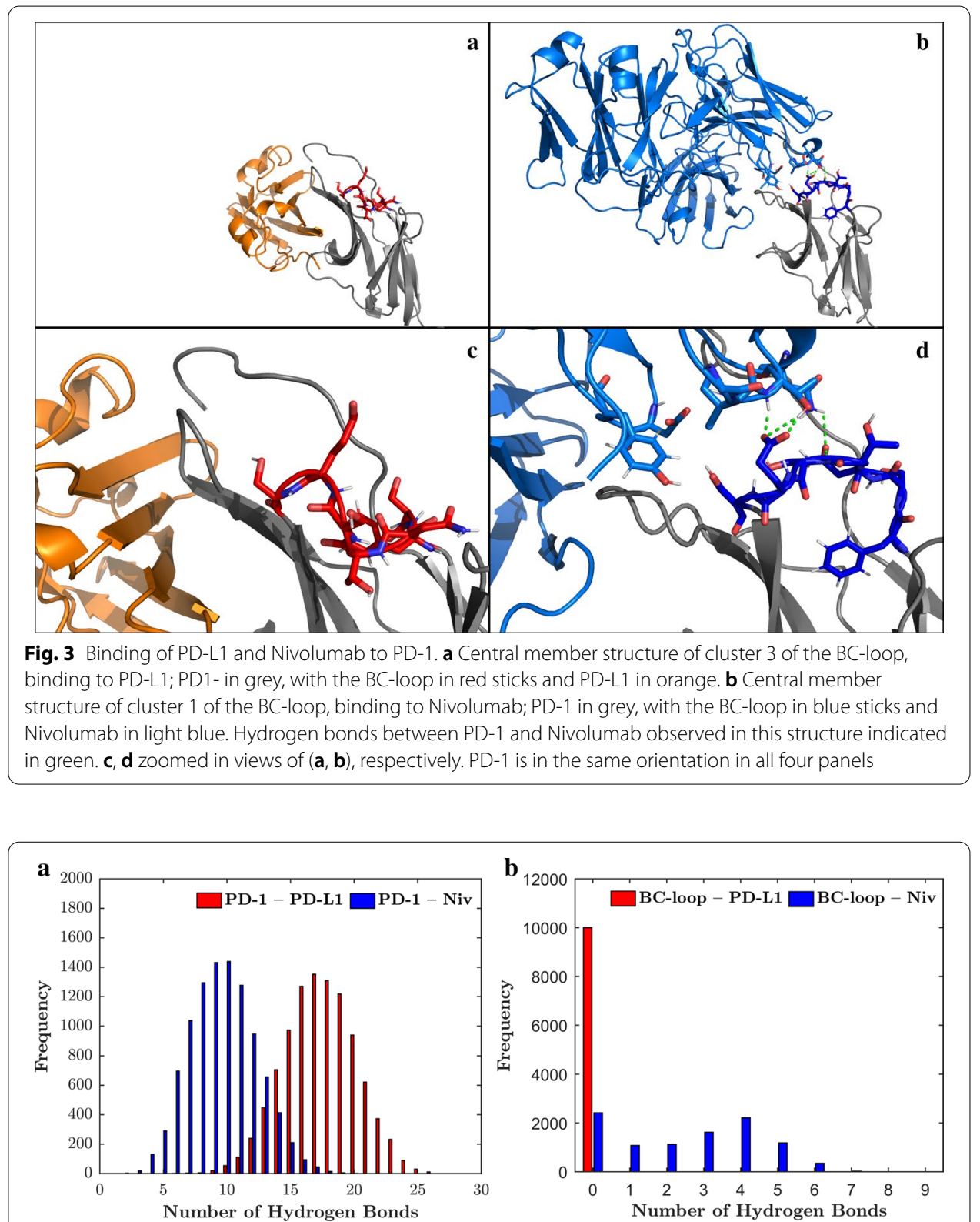

Fig. 4 The BC-loop does not form any hydrogen bonds with PD-L1. a The frequencies of the number of hydrogen bonds formed between PD-1 and PD-L1 (red) and PD-1 and nivolumab (blue) over the course of 100 ns are shown. The PD-1 - PD-L1 complex has a peak of 17 formed hydrogen bonds which occur in 1400 frames (which corresponds to $14 \mathrm{~ns}$ ). The PD-1 - Nivolumab complex formed 10 hydrogen bonds for 1500 frames. $\mathbf{b}$ Between the BC-loop and PD-L1 no hydrogen bonds are formed. The BC-loop and nivolumab form 2200 times 4 hydrogen bonds over the course of 100 ns. Also, for 2300 frames no hydrogen bond occurs between the BC-loop and nivolumab

conformation which is not exhibited in PD- $1_{\mathrm{Apo}}$. Analysis of the PD-1 $1_{\mathrm{PD}-\mathrm{L} 1}$ trajectory has shown that after $70 \mathrm{~ns}$ the BC-loop switches to conformation III (Fig. 3).

Hydrogen bond and non-bonded interaction energy analyses were done to examine potential reasons for the conformational change of the BC-loop upon PD-L1 binding and are shown as histograms (Figs. 4 and 5). Overall PD-1 forms more hydrogen bonds 

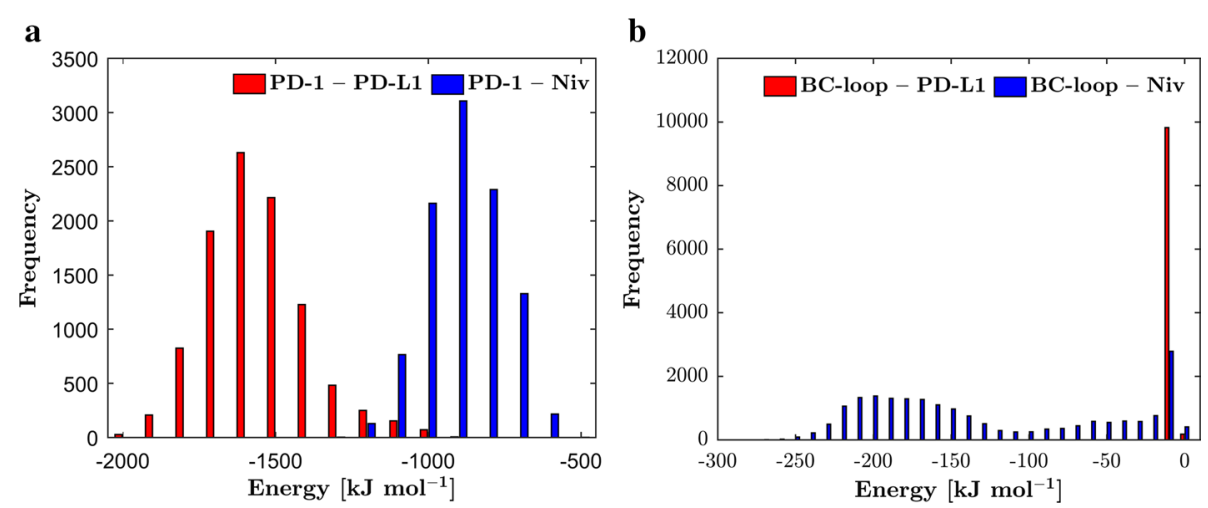

Fig. 5 No non-bonded interactions between the BC-loop and PD-L1. a The non-bonded interaction energies between PD-1 and PD-L1 (red) and PD-1 and nivolumab (blue) are shown as a histogram and are grouped in packages of $100 \mathrm{~kJ} \mathrm{~mol}^{-1}$. The non-bonded interaction energy between PD-1 and PD-L1 ranges from -1000 to $-2000 \mathrm{~kJ} \mathrm{~mol}^{-1}$ with a peak of 2700 frames at $-1600 \mathrm{~kJ} \mathrm{~mol}^{-1}$. The energy distribution between PD-1 and nivolumab ranges from -500 to $-1200 \mathrm{~kJ} \mathrm{~mol}^{-1}$. The peak is at $-900 \mathrm{~kJ} \mathrm{~mol}^{-1}$ with 3100 occurrences. $\mathbf{b}$ The non-interaction energies between the BC-loop and two ligands is grouped into packages of $10 \mathrm{~kJ} \mathrm{~mol}^{-1}$. The energy between the $\mathrm{BC}$-loop and PD-L1 peaks at- $10 \mathrm{~kJ} \mathrm{~mol}^{-1}$. This peak occurs due to the minor distortions (between 0 and $-1 \mathrm{~kJ} \mathrm{~mol}^{-1}$ ). The non-bonded interaction energy between the BC-loop and nivolumab ranges from 0 to $-250 \mathrm{~kJ} \mathrm{~mol}^{-1}$

with PD-L1 (peak at 17 hydrogen bonds) than nivolumab (10 hydrogen bonds) over the course of $100 \mathrm{~ns}$ (Fig. 4a). However, no hydrogen bonds are formed between the BCloop and PD-L1 (Fig. 4b). Nivolumab on the other hand forms up to 6 hydrogen bonds with the BC-loop during $100 \mathrm{~ns}$. Coherently, the non-bonded interaction energies show a similar pattern. The BC-loop does not interact with PD-L1 via VdW or electrostatic forces. The non-bonded interaction energy between the BC-loop and nivolumab peaks at $-120 \mathrm{~kJ} \mathrm{~mol}^{-1}$. The results corroborate with the fact that no interactions between the BC-loop and PD-L1 have been mentioned in the literature so far. Indeed, visualization of the central frame of cluster 3 (Fig. 3a and c) verify that interaction between the BC-loop and PD-L1 is spatial not possible. For nivolumab on the hand evidence of such interaction have been reported. Again, visualization of the central frame of cluster 1 shows that hydrogen bonds between the BC-loop and nivolumab are formed (Fig. 3b and d).

In a crystallization experiment Lee et al. [25] concluded (referring to Zak et al. [10]) that nivolumab induces a conformation in the BC-loop which is incompatible with PD-L1. Based on the results of the MD simulation we cannot confirm this. Admittedly, nivolumab induces a conformational change in the $\mathrm{BC}$-loop however, this conformation is compatible with PD-L1. In fact, the BC-loop shares this conformation when bound to PD-L1 for $25 \%$ of the time. In another crystallization experiment it has been described that the $\mathrm{BC}$-loop is shifted $\sim 5.3 \AA$ away when it binds to nivolumab compared to PD-L1 [22]. These findings could be confirmed as indeed, $30 \%$ of the time the $\mathrm{BC}$ loop of PD- $1_{\mathrm{PD}-\mathrm{L} 1}$ is shifted away from the BC-loop of PD- $1_{\mathrm{Niv}}$. As hydrogen bonds and non-bonded interactions are not the driver for the conformational changes, we assume entropic causes. To investigate this we suggest free energy calculations [10]. 


\section{Conclusion}

The immune checkpoint receptor PD-1 has been identified as a key target in cancer immunotherapy. The PD-1 blocking antibody nivolumab which inhibits the PD-L1 binding was recently approved by the FDA. Even though PD-1 is already used as a drug target the exact mechanism of the receptor is still unknown. Here we present the results of the first MD simulations of PD-1 with a complete extracellular domain and a focus on the role of the BC-loop of PD-1 upon binding PD-L1 or nivolumab. Visualization of the structures proofs that the combination of Daura et al. [20] clustering and multidimensional scaling is a valid approach to identify conformations. The BC-loop of PD-1 can exhibit three conformations and occurrence of the conformations depends on the binding partner. Furthermore, we identified that upon nivolumab binding the $\mathrm{BC}$-loop changes the conformation as described in the conformational selection model. PD-L1, on the other hand, does not even directly interact with the BC-loop but it nonetheless induces a conformational change in the BC-loop. This allosteric effect could play a crucial role in the activation of the PD-1 receptor.

In the future we will search for movement patterns of PD-1 based on unsupervised numerical methods and evidence from the literature.

\section{Supplementary information}

Supplementary information accompanies this paper at https://doi.org/10.1186/s12859-020-03904-9.

Additional file 1: Supplement Figure 1. Comparison of cut-offs. For the clustering algorithm it is necessary to define a cut-off (in nm). Structures within the cutoff are seen as similar and are grouped. On the one hand the cut-off must be small enough to distinguish between conformations structurally different. On the other hand, it must not bet too small to avoid over-differentiation. (A) When the cut-off is set to $0.3 \mathrm{~nm}$ five clusters are found. (B) 25 clusters are found when the cut-off is set to $0.2 \mathrm{~nm}$. (C) Over 100 clusters are found with a cut-off of $0.1 \mathrm{~nm}$.

Additional file 2: Supplement Figure 2. Incremental clustering indicates convergence of the simulation. Clustering was performed for the PD-1 ${ }_{\text {Apo }}$ simulation with a constant cut-off of $0.2 \mathrm{~nm}$ for sub-trajectories of the first $10 \mathrm{~ns}, 20 \mathrm{~ns}$ etc., see horizontal axis (time). With increasing length of sub-trajectory the number of clusters increases (vertical axis) until it finally levels off at $70 \mathrm{~ns}$. This indicates sufficient sampling allover configuration space and convergence of the simulation.

\section{Abbreviations}

APC: Antigen-presenting cells; CHI: Classical Hodgkin lymphoma; LJ: Lennard-Jones; MD: Molecular dynamics; MHC: Major histocompatibility complex; NSCLC: Non-small-cell lung carcinoma; PD-1: Programmed cell death protein I; PD-L1: Programmed cell death protein I ligand I; RMSD: Root-mean-square deviation; RMSF: Root-mean-square fluctuation; TCR: T cell receptor; VdW: Van der Waals; VSC: Vienna Scientific Cluster.

\section{Acknowledgements}

The computational results presented have been achieved using the Vienna Scientific Cluster (VSC). We thank Michael Kenn for his mathematical advice.

\section{About this supplement}

This article has been published as part of BMC Bioinformatics Volume 21 Supplement 17 2020: Selected papers from the 3rd International Workshop on Computational Methods for the Immune System Function (CMISF 2019). The full contents of the supplement are available at https://bmcbioinformatics.biomedcentral.com/articles/supplements/volum e-21-supplement-17.

\section{Authors' contributions}

B.R. performed the simulations and wrote the manuscript. C.O. contributed to select methods and algorithms. W.S. initiated, organized and supervised study. All authors read and approved the final manuscript.

\section{Funding}

There was no financial support used for this project. Publication costs are funded by the Medical University of Vienna.

\section{Availability of data and materials}

The datasets used and/or analysed during the current study are available from the corresponding author on reasonable request. 
Ethics approval and consent to participate

Not applicable.

\section{Consent for publication}

All authors read and approved the final manuscript.

\section{Competing interests}

The authors declare that they have no competing interests.

\section{Author details}

${ }^{1}$ Institute of Biosimulation and Bioinformatics, Medical University of Vienna, Spitalgasse 23/88.04.510, 1090 Vienna, Austria. ${ }^{2}$ Institute of Molecular Modeling and Simulation, University of Natural Resources and Life Science, Vienna, Muthgasse 18, 1190 Vienna, Austria.

Received: 18 November 2020 Accepted: 24 November 2020

Published: 14 December 2020

\section{References}

1. Zak KM, Grudnik P, Magiera K, Dömling A, Dubin G, Holak TA. Structural biology of the immune checkpoint receptor PD-1 and its ligands PD-L1/PD-L2. Structure. 2017;25(8):1163-74.

2. Arasanz H, Gato-Cañas M, Zuazo M, Ibañez-Vea M, Breckpot K, Kochan G, Escors D. PD1 signal transduction pathways in T cells. Oncotarget. 2017:8(31):51936-45.

3. Shen X, Zhang L, Li J, Li Y, Wang Y, Xu ZX. Recent findings in the regulation of programmed death ligand 1 expression. Front Immunol. 2019;10(1664-3224 (Electronic)):1-25.

4. Sharma P, Allison JP. The future of immune checkpoint therapy. Science. 2015;348(6230):56-61.

5. Chen L, Han X. Anti-PD-1/PD-L1 therapy of human cancer: past, present, and future. J Clin Invest. 2015;125(9):3384-91.

6. Ahmed M, Barakat K. When theory meets experiment: the PD-1 challenge. J Mol Model. 2017;23(11):308.

7. Nussinov R, Jang H, Tsai CJ, Cheng F. Review: precision medicine and driver mutations: computational methods, functional assays and conformational principles for interpreting cancer drivers. PLoS Comput Biol. 2019;15(3):e1006658.

8. Shi D, Zhou S, Liu X, Zhao C, Liu H, Yao X. Understanding the structural and energetic basis of PD-1 and monoclonal antibodies bound to PD-L1: a molecular modeling perspective. Biochim Biophys Acta Gen Subj. 2018;1862(3):576-88.

9. Feng T, Barakat K. Molecular dynamics simulation and prediction of druggable binding sites. Methods Mol Biol. 2018;1762:87-103.

10. Zak KM, Kitel R, Przetocka S, Golik P, Guzik K, Musielak B, Dömling A, Dubin G, HolakTA. Structure of the complex of human programmed death 1, PD-1, and its ligand PD-L1. Structure. 2015;23(12):2341-8.

11. Liu W, Huang B, Kuang Y, Liu G. Molecular dynamics simulations elucidate conformational selection and induced fit mechanisms in the binding of PD-1 and PD-L1. Mol BioSyst. 2017;13(5):892-900.

12. Du J, Qin Y, Wu Y, Zhao W, Zhai W, Qi Y, Wang C, Gao Y. The design of high affinity human PD-1 mutants by using molecular dynamics simulations (MD). Cell Commun Signal. 2018;16(1):25-41.

13. Pascolutti R, Sun XG, Kao J, Maute RL, Ring AM, Bowman G, Kruse AC. Structure and dynamics of PD-L1 and an ultrahigh-affinity PD-1 receptor mutant. Structure. 2016:24(10):1-10.

14. Ahmed M, Barakat $K$. The too many faces of PD-L1: a comprehensive conformational analysis study. Biochemistry. 2017;56(40):5428-39.

15. Almahmoud S, Zhong HA. Molecular modeling studies on the binding mode of the PD-1/PD-L1 complex inhibitors. Int J Mol Sci. 2019;20(18):4654.

16. Roither B, Oostenbrink C, Schreiner W. Molecular dynamics of the immune checkpoint programmed cell death protein I, PD-1: conformational changes of the BC-loop upon binding of the ligand PD-L1 and the monoclonal antibody nivolumab. In: 2019 IEEE international conference on bioinformatics and biomedicine (BIBM): 18-21 Nov. 2019; 2019. p. 2192-6.

17. Berendsen HJ, van der Spoel D, van Drunen R. GROMACS: a message-passing parallel molecular dynamics implementation. Comput Phys Commun. 1995;91:43-56.

18. Schmid N, Eichenberger AP, Choutko A, Riniker S, Winger M, Mark AE, van Gunsteren WF. Definition and testing of the GROMOS force-field versions 54A7 and 54B7. Eur Biophys J. 2011;40(7):843-56.

19. Berendsen HJC, Postma JPM, van Gunsteren WF, Hermans J. Interaction models for water in relation to protein hydration. In: Pullman B, editor. Intermolecular forces: proceedings of the fourteenth Jerusalem symposium on quantum chemistry and biochemistry held in Jerusalem, Israel, April 13-16, 1981. Dordrecht: Springer Netherlands; 1981. p. 331-42

20. Daura X, van Gunsteren WF, Mark AE. Folding-unfolding thermodynamics of a $\beta$-Heptapeptide from equilibrium simulations. Proteins. 1999:34(3):269-80.

21. Kruskal JB. Multidimensional scaling by optimizing goodness of fit to a nonmetric hypothesis. Psychometrika. 1964;29(1):1-27.

22. Tan S, Zhang H, Chai Y, Song H, Tong Z, Wang Q, Qi J, Wong G, Zhu X, Liu WJ, et al. An unexpected N-terminal loop in PD-1 dominates binding by nivolumab. Nat Commun. 2017:8:14369-79.

23. Thayer KM, Lakhani B, Beveridge DL. Molecular dynamics-Markov state model of protein ligand binding and Allostery in CRIB-PDZ: conformational selection and induced fit. J Phys Chem B. 2017;121(22):5509-14. 
24. Buehner M, Ford GC, Moras D, Olsen KW, Rossman MG. D-glyceraldehyde-3-phosphate dehydrogenase: threedimensional structure and evolutionary significance. Proc Natl Acad Sci U S A. 1973;70(11):3052-4.

25. Lee JY, Lee HT, Shin W, Chae J, Choi J, Kim SH, Lim H, Won-Heo T, Park KY, Lee YJ, et al. Structural basis of checkpoint blockade by monoclonal antibodies in cancer immunotherapy. Nat Commun. 2016;7:13354-64.

\section{Publisher's Note}

Springer Nature remains neutral with regard to jurisdictional claims in published maps and institutional affiliations.

- fast, convenient online submission

- thorough peer review by experienced researchers in your field

- rapid publication on acceptance

- support for research data, including large and complex data types

- gold Open Access which fosters wider collaboration and increased citations

- maximum visibility for your research: over $100 \mathrm{M}$ website views per year

At BMC, research is always in progress.

Learn more biomedcentral.com/submissions 